\title{
Generating stereoscopic displays with Eye Lines: Applications in instruction and research
}

\author{
STEPHEN A. WURST \\ State University of New York, Oswego, New York
}

\begin{abstract}
Eye Lines, a set of software programs for designing and running experiments using the method of adjustment, was used to generate stereoscopic stimuli. To increase the understanding of stereopsis in undergraduate instruction, students created stereograms by using Eye Lines and also viewed an Eye Lines-generated anaglyph of a Necker cube. By adjusting the amount of disparity in the anaglyph, the students noted the corresponding percept changes. The potential applications of Eye Lines to research on stereoscopic versions of illusions and on cue competition are discussed.
\end{abstract}

Stereopsis, the ability to perceive depth from fusing disparate binocular images, is a major topic in visual perception. From an instructional standpoint, students tend to be interested in stereopsis because of their familiarity with its applications, especially in ViewMasters and threedimensional movies. In my perception course, I typically have them view a variety of stereograms. However, a deeper understanding of stereopsis could be obtained if the students were to construct their own stereograms. One solution would be to instruct the students to draw stereograms to evaluate their understanding of stereopsis. A more contemporary approach would be for them to generate stereograms on a computer. These displays would have the advantage of adjusting the amount and direction of the disparity, as well as increasing the students' computer skills. The software for this activity should be easy to use, even for a novice computer user, and should allow the student to be able to adjust the amount of disparity. On our campus, and others, the cost needs to be affordable as well.

Software that meets these criteria is Eye Lines (Beagley, 1990). This software was designed for conducting visual perception experiments, especially those using the method of adjustment, and it can be used on IBM-compatible and Macintosh computers. As opposed to software such as the Active Eye and MEL student versions, Eye Lines is particularly suitable for this task, since users can construct any line stimulus they want, can have control over what segments can be adjusted, and therefore can tailor the stimuli to their research.

The preceding introduction focuses on the instructional aspects of stereopsis, but a similar problem confronts researchers. Using Eye Lines to generate stereoscopic displays and experiments is a low-cost alternative to more sophisticated and expensive software and hardware systems.

The author acknowledges the assistance of David Sargent and Wally Beagley for their helpful comments on early drafts of this article, Jim Ford for assistance with the graphics, Jennifer Gerhold for data collection and assisting students in the laboratory sessions, and Peter Hornby and Margaret Anderson for encouraging me to explore the applications of Eye Lines. Requests for reprints should be sent to $S$. A. Wurst, Department of Psychology, SUNY-College at Oswego, Oswego, NY 13126.

\section{The Eye Lines Software and Stereoscopic Displays}

The Eye Lines software consists of four programs: EL draw, EL set, EL run, and EL sort (Beagley, 1991). EL draw allows the user to create the stimuli to be used in the experiment. EL set lets the user set up the experiment by specifying which segments are to be adjusted by the subject, as well as other parameters of the experiment. EL run runs the experiment as specified by the user in EL set. EL sort allows the user to sort the subjects' data and to compute means. Because the focus of this paper is the creation of the stimuli, EL draw will be discussed here as the program of the greatest importance.

Stimuli can be created in EL draw in different modes. In Keydraw mode, the user specifies the coordinates of the line segments; in Mousedraw and Mouseline modes, the user can use the mouse as a drawing tool. The two mouse modes are appropriate if curved segments are desired, but the precision is better with Keydraw, which will be the mode discussed in detail here.

In the Keydraw mode, the user inputs on a spreadsheet the coordinates of the starting point and the end point of the line, or can specify the starting-point coordinates and the length and angle of the line. An additional column can be used to manipulate the thickness or color of the line, depending on whether Macintosh or an IBM-compatible computer is being used. Another option is to use link segments: Instead of inputting the coordinates of each segment, the user can join a new line to a previous line segment at its starting point, endpoint, or midpoint. An example of the spreadsheet, with the corresponding stimulus, is presented in Figure 1. Each row represents a different line segment; the columns specify the $x$ and $y$ starting point, the line length, the angle of the line, and the $x$ and $y$ end point. The units on the $x$-axis run from 0 to 10,000 , and those on the $y$-axis run from 0 to 7,200 (although this is machine dependent). Notice in Figure 1 that Line Segment 4 makes use of a link segment; the " $M$ " and the " 3 " in the first two columns denote that this segment should start at the midpoint of Line Segment 3.

There are various features that are included in EL draw that are helpful to the user. First, the user sees the stimulus on the screen after each line is entered into the spread- 
sheet, and therefore gets immediate feedback on the construction of the stimulus and can easily make the necessary corrections. The Preview feature also allows the user to see the stimulus without the spreadsheet and menu visible. A second feature displays the axes on the screen with tick marks at each 1,000 units, which helps the user locate the desired coordinates to input. A third feature, called Blinkseg, is particularly helpful with more complicated stimuli. The user specifies a line on the spreadsheet, and then the corresponding line in the display blinks on and off. More detailed descriptions of the various features of Eye Lines can be found in Beagley (1993).

Stereograms can be constructed in various ways (e.g., see Frisby, 1980; Rock, 1984, for more detailed descriptions). To create stereoscopic images with Eye Lines, the user can create the same image on different sides of the screen and make the position of lines on one of the images adjustable to increase the amount of disparity. Many adjustments in Eye Lines are length and angle adjustments, but position can be adjusted by linking a blank line to the line that is to be moved. For example, Figure 1 is a stereoscopic version of the inverted-T illusion. Line 2 , in this example, would need be linked to a prior blank line to adjust the lateral position of the vertical line in the stimulus on the right. Even without including an adjustable line, static stereograms can be created by adding disparity to one of the two images.

The hardware for viewing the images need not be expensive; a simple cardboard baffle placed between the two images is sufficient. Other possibilities include stereo viewers, such as the Taylor-Merchant stereopticon (Model 707, as found in Rock, 1984), and a telestereoscope, consisting of four small mirrors (e.g., Dosher, Sperling, \& Wurst, 1986). Another alternative is to create overlapping images of different colors and view this anaglyph through 3-D glasses made from colored filters (e.g., Edmund Scientific, Model N60,403) that match the colors available on your system. The size and the distance between the images need to be tailored to your viewing system.

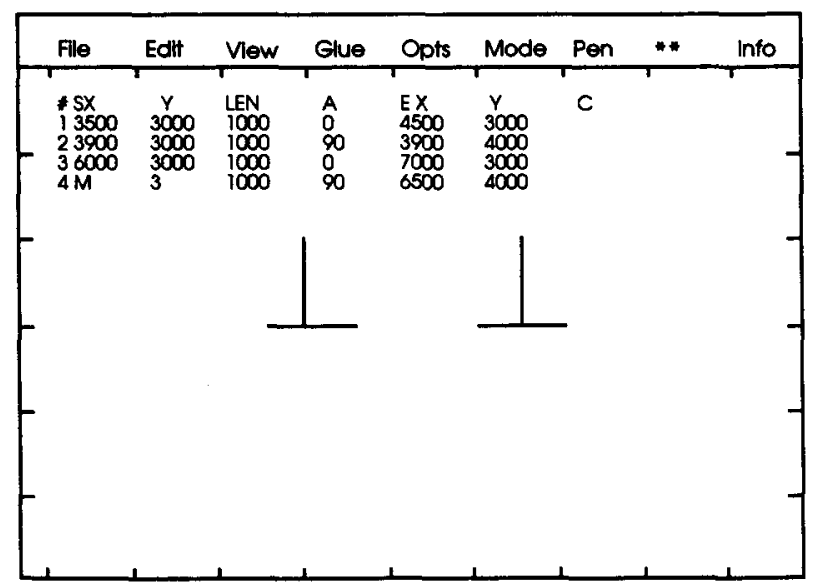

Figure 1. The construction of a stereoscopic version of the inverted$T$ illusion by using the Keydraw mode of EL draw. The top part of the screen displays the input to the spreadsheet; the bottom section shows the corresponding image created by this input.

\section{Instructional Applications}

I used Eye Lines in an undergraduate perception course to illustrate the major principles of stereopsis. After the students had read the section of their text on binocular disparity, I lectured briefly on the topic and circulated a stereogram (Keystone Eye Comfort and Depth Perception Series EC4-V32932) for them to view. I also briefly described the Keydraw mode of EL draw, in a manner similar to the description above. The students then went to the computer lab to perform two exercises. They ran an anaglyph that I had created and noticed the percept changes as they adjusted the position of one of the stimuli. They also were instructed to construct their own stereograms. The students worked in groups of between 3 and 6 , depending on the number of available computers.

The anaglyph that was presented to the students was a Necker cube drawn with linear perspective (similar to Figure 173 in Frisby, 1980, p. 77). There was an inner, smaller face and an outer, larger face. If the smaller face is perceived as being closer, the form would look like a truncated pyramid; if the larger face is perceived as being closer, the form would look like an elongated chute. The students were encouraged to look at the anaglyph through the colored lenses and move the mouse to adjust the position of the inner face of the blue image. They discovered how increasing the disparity increased the perception of depth, and how the direction of the disparity influenced one percept over the other. The students also noticed the change in percepts as they reversed the lenses to view the anaglyph. By adjusting the position of the inner face, they realized how little disparity is needed to perceive depth, and how making the disparity too great would result in the inability to fuse the two images. These last two points were particularly important because they led to a discussion of stereoacuity, Panum's area, and diplopia. Anecdotally, the success of this anaglyph was demonstrated by 2 students who spontaneously reached out about 8 in. above the screen and tried to touch the top of the "truncated pyramid."

Because the laboratory session was $1 \mathrm{~h}$ long, I believed that it would be beneficial to just have the students construct static stereograms by using EL draw, and not add the next step of incorporating blank lines to adjust position. The rationale was to introduce the software in this manner and hopefully pique the interest of the students who wanted to use the software for independent projects. To facilitate the construction of their stereograms, I provided fixation frames that could be fused with available stereo viewers. This also allowed the students to see the coordinate system. The students were instructed to introduce disparity between the two images so that some forms would look like they were closer to the observer and some would look distant to the observer. All the groups were able to construct the stereograms within $45 \mathrm{~min}$, and were able to observe the stimuli in depth. Two examples of the stereograms created by the students are presented in Figures 2A and 2B.

To evaluate the success of using Eye Lines in this laboratory, the students were asked to rate various aspects of the 
exercise on 7-point rating scales. The students $(n=22)$ first rated how easy Eye Lines was to use $(1=$ very easy; $7=$ very difficult). The results showed that they had found Eye Lines to be relatively easy to use $(M=2.91, S D=0.85)$. The students also tended to agree that the exercises had increased their understanding of stereopsis $(M=2.65, S D=$ 0.87 ; with a rating of 1 indicating strongly agree and 7 indicating strongly disagree). The students also rated the various features of Keydraw on how helpful the feature had been ( $1=$ very helpful $; 7=$ not very helpful $)$. In order of helpfulness, Preview $(M=1.80, S D=1.14)$, Blinkseg $(M=$ $2.34, S D=0.96)$, and Axes ON $(M=2.68, S D=1.05)$ all seemed to aid stimulus construction. The success of using Eye Lines was also evident when 2 of the students approached me after lab and asked if they could use Eye Lines for their required research projects.

\section{Potential Research Applications}

The advantages of Eye Lines, such as cost and ease of use, can be important to vision researchers also. To demonstrate the potential for researchers, I will describe two possible applications: (1) depth cues in conflict, and (2) stereoscopic versions of popular illusions.

In addition to binocular disparity cues, depth perception is influenced by many monocular cues. By manipulating two cues so that they conflict, a greater understanding of depth perception will result. For example, Dosher et al. (1986) pitted binocular disparity cues against a brightness cue (proximity luminance covariance; bright lines are perceived as being closer) to investigate the relative contribution of each cue on depth perception. Both static and dynamic, rotating Necker cubes were used. A workable, although less sophisticated, version of this experiment could be conducted with Eye Lines. Instead of brightness, line thickness could be used to bias one percept or the other, and put in conflict with stereopsis. The method of adjustment could then be used to see how much disparity is needed to result in different percepts.

A second example is investigating stereoscopic versions of common illusions. Stereoscopic versions of illusions, such as the Müller-Lyer, Zöllner, and Poggendorff, have been studied by Lau (1922) and Squires (1956). More recently, Gyoba (1987) examined a stereoscopic Poggendorff illusion, and Gillam (1971) proposed a depthprocessing explanation of this illusion. Other illusions that can also be easily constructed by using Eye Lines are the inverted-T (or horizontal-vertical) illusion (shown in Figure 1) and Gillam's (1980) version of the Ponzo illusion. In all of these cases, the method of adjustment (e.g., for the Poggendorff, subjects adjust the oblique until the lines are aligned) would then be used to obtain a measure of the illusion under the experimental and control conditions.

\section{Summary}

Eye Lines is an effective, affordable, and easy way to generate and display stereographic images for instructional and research purposes. It is flexible enough to generate a variety of stimuli that can be adjusted for the amount
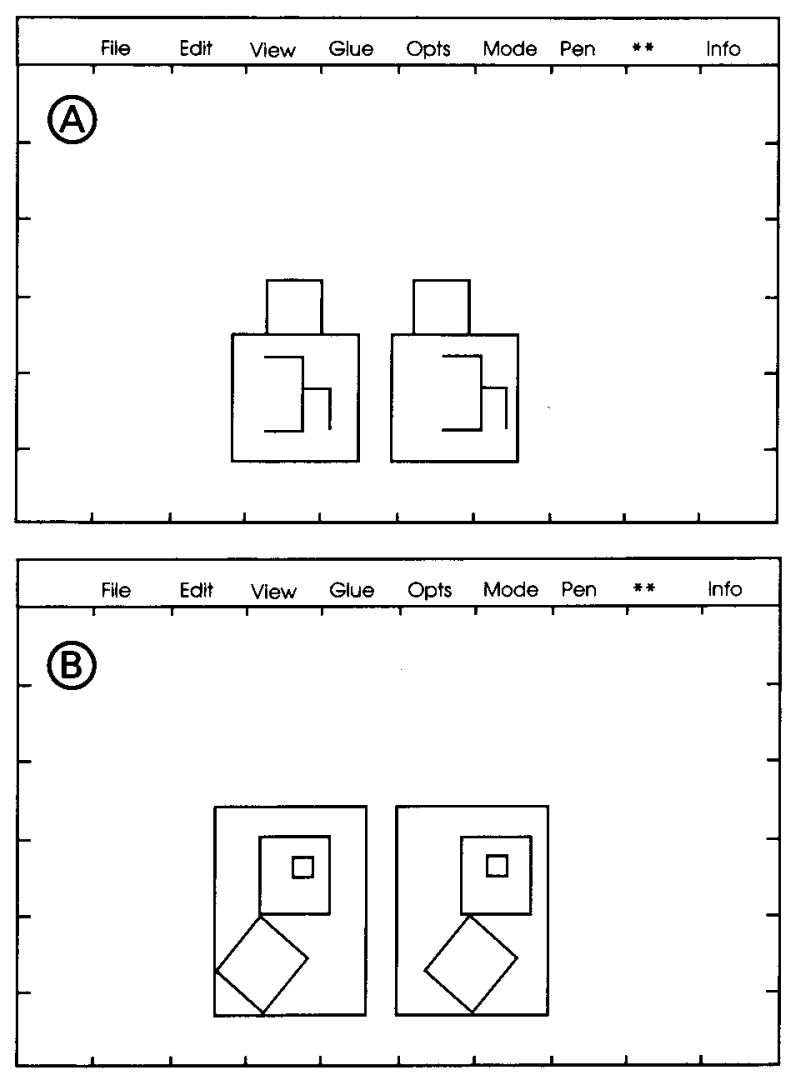

Figure 2. Two stereograms created by undergraduate students using the Keydraw mode of EL draw.

of disparity, and it can be viewed through inexpensive hardware.

\section{REFERENCES}

BEAGleY, W. K. (1990). Eye Lines [Computer program]. Alma, MI: Alma College.

BEAGLEY, W. K. (1991). EYE LINES: A tool for studying visual perception of size and angle. Behavior Research Methods, Instruments, \& Computers, 23, 183-186.

BEAGLEY, W. K. (1993). Eye Lines: Generating data through image manipulation, issues in interface design, and the teaching of experimental thinking. Behavior Research Methods, Instruments, \& Computers, 25, 333-336.

Dosher, B. A., SPerling, G., \& Wurst, S. A. (1986). Tradeoffs between stereopsis and proximity luminance covariance as determinants of perceived 3D structure. Vision Research, 26, 973-990.

FrISBY, J. P. (1980). Seeing: Illusion, brain, and mind. Oxford: Oxford University Press.

GiLlam, B. (1971). A depth processing theory of the Poggendorff illusion. Perception \& Psychophysics, 10, 211-216.

Gillam, B. (1980). Geometrical illusions. Scientific American, 242, $102-111$.

Groba, J. (1978). The Poggendorff illusion under stereopsis. Tohuku Psychologica Folia, 37, 94-101. (From PsycLit database, Abstract No. 64-07041)

LAU, E. (1922). Über das stereoskopische Sehen. Psychologische Forschung, 2, 1-5.

Rock, I. (1984). Perception. New York: W.H. Freeman.

SQUIRES, P. C. (1956). Stereopsis produced without horizontally disparate stimulus loci. Journal of Experimental Psychology, 52, 199-203. 